\title{
SVIR Epidemic Model with Non Constant Population
}

\author{
Joko Harianto1, Titik Suparwati² \\ 1,2Mathematics Department, Cenderawasih University \\ Email: joharijpr88@gmail.com
}

\begin{abstract}
In this article, we present an SVIR epidemic model with deadly deseases and non constant population. We only discuss the local stability analysis of the model. Initially the basic formulation of the model is presented. Two equilibrium point exists for the system; disease free and endemic equilibrium point. The local stability of the disease free and endemic equilibrium exists when the basic reproduction number less or greater than unity, respectively. If the value of $R_{0}$ less than one then the desease free equilibrium point is locally asymptotically stable, and if its exceeds, the endemic equilibrium point is locally asymptotically stable. The numerical results are presented for illustration.
\end{abstract}

Keywords: SVIR Model, Basic Reproduction Number, Numerical results

\section{INTRODUCTION}

Health problems are closely associated with a disease. It is motivating humans to study health as well as illness. One of the goals of a nation to achieve prosperity in the future is the creation of a healthy living environment. One of the indicators of a healthy environment involves a high level of quality health services. Quality health care issues need to be addressed systematically, effectively and efficiently. Analysis of the spread of a disease is one step to address the problem of quality health services.

The spread of disease due to virus or bacteria that enter into the body will lead to human health disorders and will affect the socio-economic development of the community. Efforts to prevent a spreading disease can work optimally if several stages such as research, development of various diagnostic tools, drugs, and new vaccines, has achieved.

Mathematical models are one of a useful tool for reviewing or analyse the patterns of a disease spread. According to [2] and [4] the spread of infectious diseases can be described mathematically through a model, such as SIR and SIRS. The most basic procedure in modelling the spread of disease is by using the compartment model. In this case, the population is divided into three different classes namely, Susceptible, Infected, and Recovered which then shortened to SIR. Vaccination can be considered as the addition of a class naturally into the epidemic base model for some types of diseases.

Along with the development of knowledge in the field of health, control of an epidemic disease can be done by vaccination action. Then the SIR model develops with the addition of a vaccinated population class.

Some researchers have proposed models of the dynamics of vaccination. Among others is SVI type, which has been developed by [5], [6] and [8]. Several studies have stated that the threshold to determine the occurrence of endemic disease seen from the value of Basic Reproduction Number $\left(R_{0}\right)$. The disease distribution model will be locally stable if $R_{0} \leq 1$ and the disease will be present if $R_{0}>1$. Paper [9] discusses the SVIR epidemic model for non-lethal diseases that does not involve the individual's mortality 
rate due to the disease. From these studies, we can build some further assumptions related to the SVIR epidemic model that developed in public life. In this article we consider local stability analysis of an SVIR epidemic model with deadly disease and non constant population based on SVIR epidemic model developed in [8]. We hope the model can be useful to analyse the disease outbreaks so that we can take optimal precautions.

\section{METHODS}

This research used literature study with literature sources from some reputable journal. The simulation of model SVIR used mathematical software, i.e. Maple 2016.

\section{RESULTS AND DISCUSSION}

The following are assumptions used in modelling:

1. Closed population (no migration)

2. Births occur in every sub populations and enter in vulnerable subpopulations.

3. Natural birth occurs on each sub populations at the same rate

4. Natural death occurs on each sub populations at the different rate.

5. Illness can cause death.

6. Individuals who have cured cannot return to susceptive class (permanent cure).

7. Short incubation period.

8. Vaccination has given to susceptible subpopulations.

9. We do not distinguish the rate of recovery for a child has been Infected and for the adult.

10. Vaccination will reach the level of immunity over time and finally enters the sub populations that heals.

11. Individuals who have vaccinated become Infected if they lose immunity.

12. Contact between Infected and susceptive individuals can lead to disease transmission, and so are contact between Infected and those have vaccinated.

We investigate the basic model formulation by dividing the total population into four compartments, such as $\mathrm{S}(\mathrm{t})$ for susceptible, $\mathrm{V}(\mathrm{t})$ for vaccinated, $\mathrm{I}(\mathrm{t})$ for Infected and $\mathrm{R}(\mathrm{t})$ for recovered individuals. Let $\beta$ is the rate of contact that is sufficient to transmit the disease. We also assume a constant recovery rate $\gamma$. The rate at which the susceptible population is vaccinated is $\alpha$. We assume that there can be disease related death and define $\omega$ to be the rate of disease related death, while $\mu$ is the rate of natural death that is not related to the disease. Let $\mu_{1}$ be the natural death rate for susceptible population, $\mu_{2}$ be the natural death rate for vaccination population, $\mu_{3}$ be the natural death rate for Infected population, $\mu_{4}$ be the natural death rate for recovery population. We assume that all newborns enter the susceptible population at the constant rate of $\mu$. Let $\gamma_{1}$ be the average rate for susceptible individuals to obtain immunity and moved into recovered population. We do not distinguish the natural immunity and vaccine-induced immunity here because vaccine-induced immunity can also last for a long term. We assume that before obtaining immunity the vaccinees still have possibility of infection with a disease transmission rate $\beta_{1}$ while contacting with Infected individuals. $\beta_{1}$ may be assume to be less than $\beta$ because the vaccinating individuals may have some partial immunity during the process or they may recognize the transmission characters of the disease and hence decrease the effective contacts with Infected individuals. Figure 1 shows the details of the population transfer diagram of the SVIR epidemic model. 


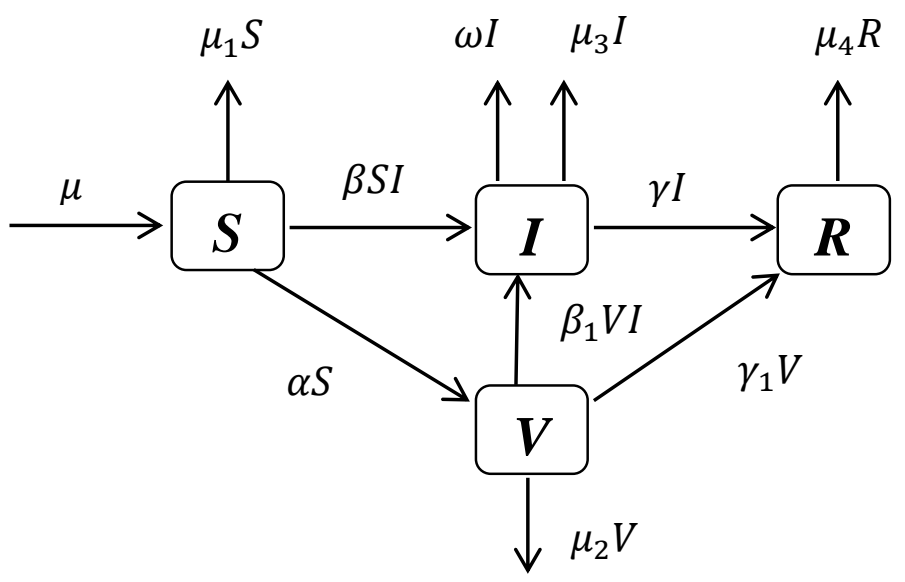

Figure 1. Transfer diagram of the SVIR epidemic model with deadly deseases and non constant population

The following form presents the system model of differential equations.

$$
\begin{aligned}
& \frac{d S}{d t}=\mu-\mu_{1} S-\beta S I-\alpha S \\
& \frac{d V}{d t}=\alpha S-\beta_{1} V I-\gamma_{1} V-\mu_{2} V \\
& \frac{d I}{d t}=\beta S I+\beta_{1} V I-\gamma I-\mu_{3} I-\omega I \\
& \frac{d R}{d t}=\gamma_{1} V+\gamma I-\mu_{4} R
\end{aligned}
$$

where $S(0)>0, V(0)>0, I(0)>0, R(0)=0$, with $S(0)+V(0)+I(0)+R(0)=1$, and

\begin{tabular}{|c|c|}
\hline Parameter & Description \\
\hline$\mu$ & Birth rate \\
\hline$\beta$ & $\begin{array}{l}\text { The transmission rate of disease when susceptible } \\
\text { individuals contact with Infected individuals }\end{array}$ \\
\hline$\alpha$ & $\begin{array}{l}\text { The rate of migration of susceptible individuals to } \\
\text { the vaccination process }\end{array}$ \\
\hline$\beta_{1}$ & $\begin{array}{l}\text { The transmission rate for vaccination individuals } \\
\text { to be infected before gaining immunity }\end{array}$ \\
\hline$\gamma_{1}$ & $\begin{array}{l}\text { The average rate for vaccination individuals to } \\
\text { obtain immunity and move into recovered } \\
\text { population }\end{array}$ \\
\hline$\omega$ & The death rate due to disease \\
\hline$\gamma$ & Recovery rate \\
\hline$\mu_{1}$ & Natural death rate of the susceptible population \\
\hline$\mu_{2}$ & Natural death rate of the vaccination population \\
\hline$\mu_{3}$ & Natural death rate of the Infected population \\
\hline$\mu_{4}$ & Natural death rate of the recovery population \\
\hline
\end{tabular}
all parameters are positive. The parameters with their description are presented in the following table.

Table 1. Parameter Description 


\section{Equilibrium Point}

As a first step, we define equilibrium points of the system (disease free equilibrium point and endemic equilibrium point). In general, according to [1] and [3] there exist two equilibrium points of epidemic model, i.e. the disease free equilibrium point and the endemic equilibrium point. The two equilibrium points are reviewed based on the existence of the disease in a population with a continuous time. The disease-free equilibrium point is the point at which the disease is unlikely to spread in an area because the Infected population is equal to zero $(I=0)$ for $t \rightarrow \infty$. While the endemic equilibrium point is the point at which the disease must spread $(I>0)$ for $t \rightarrow \infty$, in a defined closed area.

The equilibrium point of system (1) exists when

so that:

$$
\frac{d S}{d t}=0 \quad, \quad \frac{d V}{d t}=0 \quad, \quad \frac{d I}{d t}=0 \quad, \quad \frac{d R}{d t}=0
$$

$$
\begin{array}{r}
\mu-\mu_{1} S-\beta S I-\alpha S=0 \\
\alpha S-\beta_{1} V I-\gamma_{1} V-\mu_{2} V=0 \\
\beta S I+\beta_{1} V I-\gamma I-\mu_{3} I-\omega I=0 \\
\gamma_{1} V+\gamma I-\mu_{4} R=0
\end{array}
$$

From equation (4), we have $I\left(\beta S+\beta_{1} V-\gamma-\mu_{3}-\omega\right)=0$, and the solutions are $I=0$ or $\beta S+\beta_{1} V-\gamma-\mu_{3}-\omega=0$, consequently, we have to situation, namely:

a) Situation at $I=0$

It is the necessary condition for the disease-free equilibrium point. Note that,

- From equation (2), we have $\mu-\mu_{1} S-\alpha S=0 \Leftrightarrow S=\frac{\mu}{\alpha+\mu_{1}}$

- From equation (3), we have $\alpha S-\gamma_{1} V-\mu_{2} V=0 \Leftrightarrow V=\frac{\alpha S}{\left(\gamma_{1}+\mu_{2}\right)}=\frac{\alpha \mu}{\left(\alpha+\mu_{1}\right)\left(\gamma_{1}+\mu_{2}\right)}$

- From equation (5), we have $\gamma_{1} V+\gamma I-\mu_{4} R=0 \Leftrightarrow R=\frac{\gamma_{1} V}{\mu_{4}}=\frac{\gamma_{1} \alpha \mu}{\mu_{4}\left(\alpha+\mu_{1}\right)\left(\gamma_{1}+\mu_{2}\right)}$

So we get the disease-free equilibrium, that is,

$$
E_{0}=\left(S_{0}, V_{0}, I_{0}, R_{0}\right)=\left(\frac{\mu}{\alpha+\mu_{1}}, \frac{\alpha \mu}{\left(\alpha+\mu_{1}\right)\left(\gamma_{1}+\mu_{2}\right)}, 0, \frac{\gamma_{1} \alpha \mu}{\mu_{4}\left(\alpha+\mu_{1}\right)\left(\gamma_{1}+\mu_{2}\right)}\right)
$$

\section{b) Situation at $I>0$,}

This situation is a necessary condition for an endemic equilibrium point. Note that:

- From equation (2), we have $\mu-\mu_{1} S-\beta S I-\alpha S=0 \Leftrightarrow S^{*}=\frac{\boldsymbol{\mu}}{\left(\boldsymbol{\alpha}+\boldsymbol{\mu}_{\mathbf{1}}+\boldsymbol{\beta} I^{*}\right)}$

- From equation (3), we have

$$
\alpha S-\beta_{1} V I-\gamma_{1} V-\mu_{2} V=0 \Leftrightarrow V^{*}=\frac{\alpha \mu}{\left(\boldsymbol{\alpha}+\boldsymbol{\mu}_{1}+\boldsymbol{\beta I}^{*}\right)\left(\gamma_{1}+\boldsymbol{\mu}_{2}+\boldsymbol{\beta}_{1} I^{*}\right)}
$$

From equation (4), if $I \neq 0$, then $\beta S+\beta_{1} V-\gamma-\mu_{3}-\omega=0$, consequently, with substitute $S^{*}$ and $V^{*}$ we have:

$$
\begin{aligned}
& & \frac{\beta \mu}{\left(\alpha+\mu_{1}+\beta I^{*}\right)}+\frac{\beta_{1} \alpha \mu}{\left(\alpha+\mu_{1}+\beta I^{*}\right)\left(\gamma_{1}+\mu_{2}+\beta_{1} I^{*}\right)}-\left(\mu_{3}+\gamma+\omega\right) & =0 \\
\Leftrightarrow & & \left(\mu_{3}+\gamma+\omega\right)-\frac{\beta \mu}{\left(\alpha+\mu_{1}+\beta I^{*}\right)}-\frac{\alpha \mu \beta_{1}}{\left(\alpha+\mu_{1}+\beta I^{*}\right)\left(\gamma_{1}+\mu_{2}+\beta_{1} I^{*}\right)} & =0 \\
\Leftrightarrow & & \left(\mu_{3}+\gamma+\omega\right)-\frac{\beta \mu}{\left(\alpha+\mu_{1}+\beta I^{*}\right)}-\frac{\beta_{1} \alpha \mu}{k} & =0 \\
\Leftrightarrow & & \left(\mu_{3}+\gamma+\omega\right) k-\beta \mu\left(\gamma_{1}+\mu_{2}+\beta_{1} I^{*}\right)-\alpha \mu \beta_{1} & =0
\end{aligned}
$$

with $k=\left(\alpha+\mu_{1}+\beta I^{*}\right)\left(\gamma_{1}+\mu_{2}+\beta_{1} I^{*}\right)=\beta \beta_{1} I^{*}+\left[\left(\alpha+\mu_{1}\right) \beta_{1}+\left(\gamma_{1}+\mu_{2}\right) \beta\right] I^{*}-\beta_{1} \alpha \mu$ and then we have 


$$
A_{1} I^{* 2}+A_{2} I^{*}+A_{3}\left(1-\left(\frac{\beta \mu}{\left(\alpha+\mu_{1}\right)\left(\mu_{3}+\gamma+\omega\right)}+\frac{\beta_{1} \alpha \mu}{\left(\alpha+\mu_{1}\right)\left(\gamma_{1}+\mu_{2}\right)\left(\mu_{3}+\gamma+\omega\right)}\right)\right)=0
$$

where:

$$
\begin{aligned}
& A_{1}=\left(\mu_{3}+\gamma+\omega\right) \beta_{1} \beta>0 \\
& A_{2}=\left(\mu_{3}+\gamma+\omega\right)\left[\left(\alpha+\mu_{1}\right) \beta_{1}+\left(\gamma_{1}+\mu_{2}\right) \beta\right]-\beta_{1} \beta \mu \\
& A_{3}=\left(\mu_{3}+\gamma+\omega\right)(\alpha+\mu)\left(\gamma_{1}+\mu\right)>0
\end{aligned}
$$

Let

$$
C=\frac{\beta \mu}{\left(\alpha+\mu_{1}\right)\left(\mu_{3}+\gamma+\omega\right)}+\frac{\beta_{1} \alpha \mu}{\left(\alpha+\mu_{1}\right)\left(\gamma_{1}+\mu_{2}\right)\left(\mu_{3}+\gamma+\omega\right)},
$$

So, the above equation becomes:

$$
A_{1} I^{* 2}+A_{2} I^{*}+A_{3}(1-C)=0
$$

with the roots of equation (6), i.e.

$$
I_{1,2}^{*}=\frac{-A_{2} \pm \sqrt{A_{2}^{2}-4 A_{1} A_{3}(1-C)}}{2 A_{1}}
$$

In this case, the proportion of the Infected population must be positive. So, the existence of the endemic equilibrium point of Infected population depends on the value of C. From equation (6) we can have positive $\operatorname{root}\left(I^{*}>0\right)$ if $C>1$. The value of a positive endemic equilibrium point lies in the value of $\mathrm{C}$, so that it can be defined the value basic reproduction number, that is,

$$
R_{0}=\frac{\beta \mu}{\left(\alpha+\mu_{1}\right)\left(\mu_{3}+\gamma+\omega\right)}+\frac{\beta_{1} \alpha \mu}{\left(\alpha+\mu_{1}\right)\left(\gamma_{1}+\mu_{2}\right)\left(\mu_{3}+\gamma+\omega\right)}
$$

In epidemiology, the basic reproduction number shows about the disease spread and control. If $R_{0}<1$, then the disease free equilibrium is stable, the disease dies out from population. If $R_{0}>1$ the endemic equilibrium exists, and disease permanently exist in the population.

It is observed that $I^{*}$ exist if $R_{0}>1$.

Thus, we obtain an endemic equilibrium point if $R_{0}>1$, that is,

$$
E^{*}=\left(S^{*}, V^{*}, I^{*}, R^{*}\right)=\left(\frac{\mu}{\left(\alpha+\mu_{1}+\beta I^{*}\right)}, \frac{\alpha \mu}{\left(\alpha+\mu_{1}+\beta I\right)\left(\gamma_{1}+\mu_{2}+\beta_{1} I^{*}\right)}, I^{*}, \frac{\gamma_{1} V^{*}+\gamma I^{*}}{\mu_{4}}\right) \text {. }
$$

The existence of disease free equilibrium point is not depends on $R_{0}$ condition. Thus we conclude that, If $R_{0} \leq 1$, then there is exist one (unique) equilibrium of system (1), that is, the disease-free equilibrium point $E_{0}$. Furthermore, if $R_{0}>1$, then there are two equilibrium points of system (1), that is, the disease-free equilibrium point $E_{0}$ and the endemic equilibrium point $E^{*}$.

\section{Local Stability Analysis of Equilibrium Point}

We will determine the stability of the equilibrium point of the SVIR model around the disease-free equilibrium and the endemic equilibrium point. Next, we will study system dynamics in equation (1). Since the last equation does not depend on other equations, we simply study the following system.

$$
\begin{aligned}
& \frac{d S}{d t}=\mu-\mu_{1} S-\beta S I-\alpha S \\
& \frac{d V}{d t}=\alpha S-\beta_{1} V I-\gamma_{1} V-\mu_{2} V
\end{aligned}
$$




$$
\frac{d I}{d t}=\beta S I+\beta_{1} V I-\gamma I-\mu_{3} I-\omega I
$$

Furthermore, we performed a SVIR model stability analysis around the equilibrium point of the system (7). To discuss the local stability of system (7), we compute the Jacobian matrix of system (7). The signs of the real parts of the eigenvalues of the Jacobian Matrix evaluated at a given equilibrium determine its stability. The entries of general Jacobian matrix are given by differentiating the right hand side of system (7) with respect to $S, V, I$. The disease free stability and the endemic stability are presented in the following results.

\section{Theorem 1}

Defined:

$$
R_{0}=\frac{\beta \mu}{\left(\alpha+\mu_{1}\right)\left(\mu_{3}+\gamma+\omega\right)}+\frac{\alpha \beta_{1} \mu}{\left(\alpha+\mu_{1}\right)\left(\gamma_{1}+\mu_{2}\right)\left(\mu_{3}+\gamma+\omega\right)}
$$

i. If $R_{0}<1$, then the equilibrium point $E_{0}$ is locally asymptotically stable and if $R_{0}>1$, then the equilibrium point $E_{0}$ is unstable.

ii. If $R_{0}>1$, then the endemic equilibrium $E^{*}$ is locally asymptotically stable.

\section{Proof:}

(i). The Jacobian Matrix of system (2) is

$$
J(S, V, I)=\left(\begin{array}{ccc}
-\left(\alpha+\mu_{1}+\beta_{1} I\right) & 0 & -\beta S \\
\alpha & -\left(\gamma_{1}+\mu_{2}+\beta_{1} I\right) & -\beta_{1} V \\
\beta I & \beta_{1} I & \beta S+\beta_{1} V-\left(\mu_{3}+\gamma+\omega\right)
\end{array}\right)
$$

So the Jacobian matrix around the point $E_{0}=\left(S_{0}, V_{0}, I_{0}\right)=\left(\frac{\mu}{\alpha+\mu_{1}}, \frac{\alpha \mu}{\left(\alpha+\mu_{1}\right)\left(\gamma_{1}+\mu_{2}\right)}, 0\right)$ is

$$
J\left(E_{0}\right)=\left(\begin{array}{ccc}
-\left(\alpha+\mu_{1}\right) & 0 & -\beta S_{0} \\
\alpha & -\left(\gamma_{1}+\mu_{2}\right) & -\beta_{1} V_{0} \\
0 & 0 & \beta S_{0}+\beta_{1} V_{0}-\left(\mu_{3}+\gamma+\omega\right)
\end{array}\right)
$$

The characteristic equation is

$\Leftrightarrow\left[\lambda+\left(\alpha+\mu_{1}\right)\right]\left[\lambda+\left(\gamma_{1}+\mu_{2}\right)\right]\left[\beta S_{0}+\beta_{1} V_{0}-\left(\mu_{3}+\gamma+\omega\right)\right]=0$

where

$\lambda_{1}=-\left(\alpha+\mu_{1}\right)<0$

$\lambda_{2}=-\left(\gamma_{1}+\mu_{2}\right)<0$

$\lambda_{3}=\beta S_{0}+\beta_{1} V_{0}-\left(\mu_{3}+\gamma+\omega\right)=\left(\mu_{3}+\gamma+\omega\right)\left(R_{0}-1\right)$

It is clear if $R_{0}<1$ then all the eigenvalues of $J\left(E_{0}\right)$ are negative, hence the point $E_{0}$ is locally asymptotically stable. Meanwhile, if $R_{0}>1$, then there is a positive eigenvalue, hence the point $E_{0}$ is unstable.

(ii). It is clear that the endemic point $E^{*}$ exist when $R_{0}>1$, so the Jacobian matrix around the point $E^{*}=\left(S^{*}, V^{*}, I^{*}\right)$ is

$$
J\left(E^{*}\right)=\left(\begin{array}{ccc}
-\left(\alpha+\mu_{1}+\beta I^{*}\right) & 0 & -\beta S \\
\alpha & -\left(\gamma_{1}+\mu_{2}+\beta_{1} I^{*}\right) & -\beta_{1} V \\
\beta I^{*} & \beta_{1} I^{*} & \beta S^{*}+\beta_{1} V^{*}-\left(\mu_{3}+\gamma+\omega\right)
\end{array}\right)
$$

We can modify the element $a_{11}, a_{22}, a_{33}$, from matrix above into

$\alpha+\mu_{1}+\beta I^{*}=\frac{\mu}{S^{*}}$

$\gamma_{1}+\mu_{2}+\beta_{1} I^{*}=\frac{\alpha S^{*}}{V^{*}}$

Based on the evaluation results around the endemic equilibrium point we are getting $\beta S^{*}+\beta_{1} V^{*}-\mu_{3}-\gamma-\omega=0$,

and the matrix become 


$$
J\left(E^{*}\right)=\left(\begin{array}{ccc}
-\frac{\mu}{S^{*}} & 0 & -\beta S^{*} \\
\alpha & -\frac{\alpha S^{*}}{V^{*}} & -\beta_{1} V^{*} \\
\beta I^{*} & \beta_{1} I^{*} & 0
\end{array}\right)
$$

The characteristic equation of matrix $J\left(E^{*}\right)$ is

$\lambda^{3}+a_{1} \lambda^{2}+a_{2} \lambda+a_{3}=0$

where

$$
\begin{aligned}
& a_{1}=\frac{\mu}{S^{*}}+\frac{\alpha S^{*}}{V^{*}}>0 \\
& a_{2}=\frac{\alpha \mu}{V^{*}}+\beta_{1}{ }^{2} V^{*} I^{*}+\beta^{2} S^{*} I^{*}>0 \\
& a_{3}=\alpha \beta_{1} \beta S^{*} I^{*}+\frac{\alpha \beta^{2} S^{* 2} I^{*}}{V^{*}}+\frac{\mu \beta_{1}{ }^{2} V^{*} I^{*}}{S^{*}}>0
\end{aligned}
$$

hence,

$$
\begin{aligned}
a_{1} a_{2}-a_{3} & =\left(\frac{\mu}{S^{*}}+\frac{\alpha S^{*}}{V^{*}}\right)\left(\frac{\alpha \mu}{V^{*}}+\beta_{1}^{2} V^{*} I^{*}+\beta^{2} S^{*} I^{*}\right)-\left(\alpha \beta_{1} \beta S^{*} I^{*}+\frac{\alpha \beta^{2} S^{* 2} I^{*}}{V^{*}}+\frac{\mu \beta_{1}{ }^{2} V^{*} I^{*}}{S^{*}}\right) \\
& =\frac{\alpha \mu^{2}}{S^{*} V^{*}}+\mu \beta^{2} I^{*}+\frac{\alpha^{2} \mu S^{*}}{V^{* 2}}+\alpha \beta_{1}^{2} S^{*} I^{*}-\alpha \beta_{1} \beta S^{*} I^{*} \\
& =\frac{\alpha \mu^{2}}{S^{*} V^{*}}+\left(\alpha+\mu_{1}+\beta I^{*}\right) \beta^{2} S^{*} I^{*}+\frac{\alpha^{2} \mu S^{*}}{V^{* 2}}+\alpha S^{*} I^{*}\left(\beta-\beta_{1}\right)^{2}+\alpha \beta_{1} \beta S^{*} I^{*}>0 .
\end{aligned}
$$

It can be seen that all of the coefficients $a_{i}>0, i=1,2,3$ are positive. The Routh-Hurwitz criteria that are necessary and sufficient for the local asymptotic stability of the endemic equilibrium are that the coefficient are positive and the Hurwitz determinants $H_{i}$ are all positive [7]. For a third degree polynomial, the Hurwitz determinants criteria are $H_{1}=$ $a_{1}>0, H_{2}=a_{3}>0$ and $H_{3}=a_{1} a_{2}-a_{3}>0$. By Routh-Hurwitz criteria, roots of equation (7) have negative real parts if $a_{1} a_{2}-a_{3}>0$. Note that all term in $a_{1} a_{2}-a_{3}$ is always positive. Thus, the Routh-Hurwitz criteria satisfied for all parameter values. Therefore, by Routh-Hurwitz criteria the endemic equilibrium point $E^{*}=\left(S^{*}, V^{*}, I^{*}\right)$ is locally asymptotically stable.

Based on the results, we will interpret the results biologically as follows,

- If $R_{0}<1$, then for $t \rightarrow \infty$ and $(S, V, I)$ that close enough to $E_{0}=\left(S_{0}, V_{0}, I_{0}\right)$, the solution of system (1) will reach the value $E_{0}=\left(S_{0}, V_{0}, I_{0}\right)$. It means that if $R_{0}<1$, then for the number of susceptible, vaccinated and infected individuals close to $E_{0}=\left(S_{0}, V_{0}, I_{0}\right)$, the disease will not plague and tends to disappear indefinitely time. This condition is then called asymptotically stable around the equilibrium point $E_{0}=\left(S_{0}, V_{0}, I_{0}\right)$, and we interpret as a disease-free equilibrium point.

- If $R_{0}>1$, then for $t \rightarrow \infty$ and $(S, V, I)$ that close enough to $E^{*}=\left(S^{*}, V^{*}, I^{*}\right)$, the solution of system (1) will reach the value $E^{*}=\left(S^{*}, V^{*}, I^{*}\right)$. This means if $R_{0}>1$, then for the number of susceptible, vaccinated and Infected individuals close to $E^{*}=$ $\left(S^{*}, V^{*}, I^{*}\right)$, the disease will plague but does not reach extinction in an infinite time. This condition is then called asymptotically stable around the equilibrium point $E^{*}=$ $\left(S^{*}, V^{*}, I^{*}\right)$, and we interpret as an endemic equilibrium point.

\section{NUMERICAL RESULTS}

To substantiate the above analitycal findings, the model is studied numerically. We will discuss the results of the model by taking numerical example. We consider the parameters $\mu=0,03, \mu_{1}=0,04, \mu_{2}=0,035, \mu_{3}=0,06, \mu_{4}=0,045, \beta=0,5, \beta_{1}=$ $0,05, \gamma=0,1, \gamma_{1}=0,1, \alpha=0,2, \omega=0,1$ and the basic reproduction number is 
$R_{0}=0,28<1$. The diseases free equilibrium point for this set of parameters are $(0.125,0.185,0,0.41)$. The numerical results are shown in Figure 1 and 2. Figure 1, illustrate the fact, when the basic reproduction number less than unity, the infected proportion will reach to zero so that the desease free equilibrium point is locally asymptotically stable.

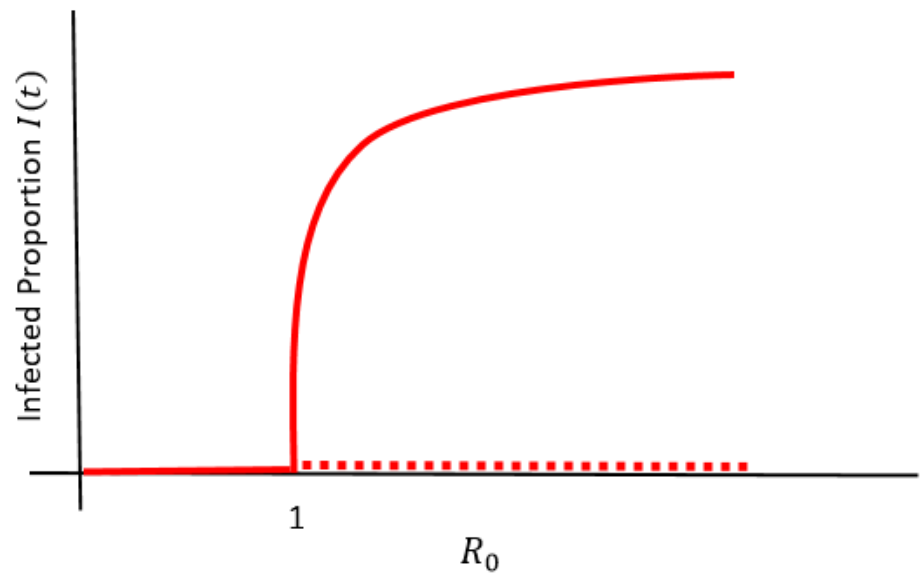

Figure 2. Basic reproduction number $R_{0}$ versus infected proportion $I(t)$

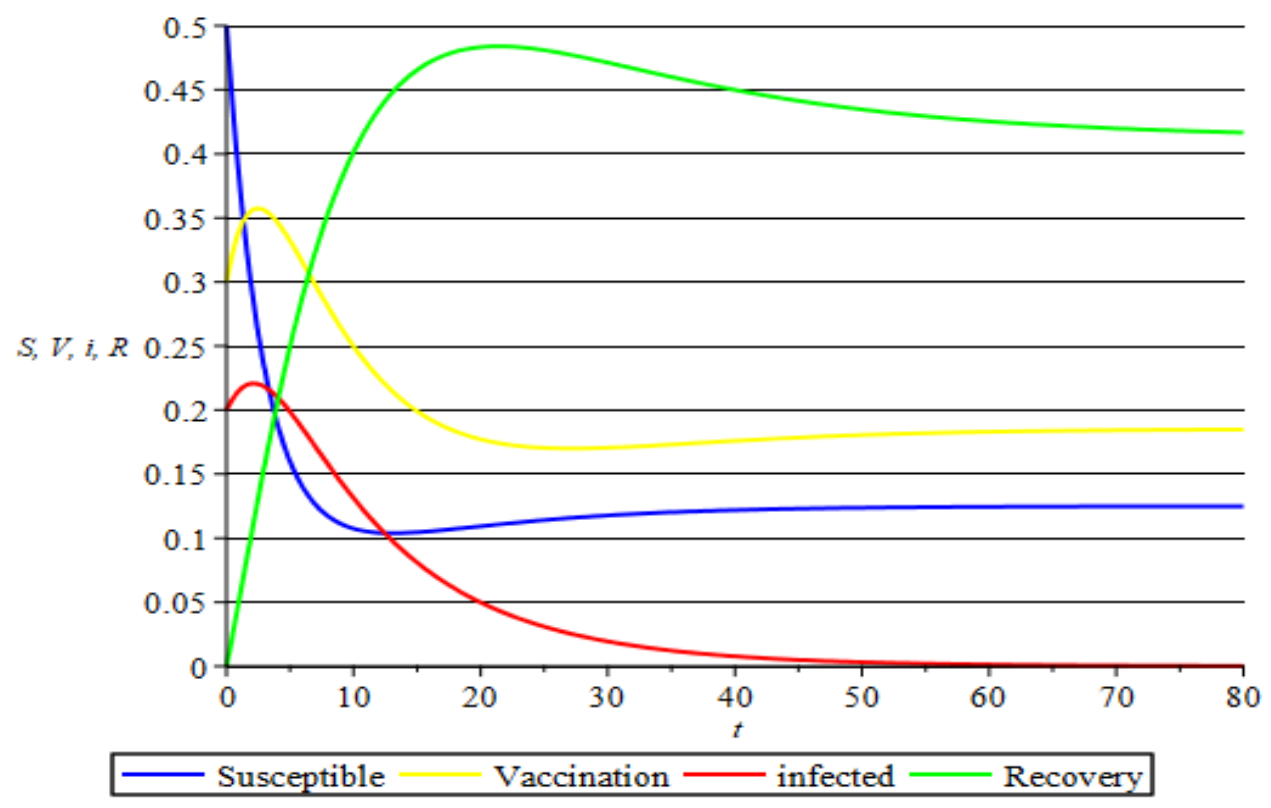

Figure 3. The dynamical behavior of system (1), when $R_{0}<1$ and the initial conditions are $S(0)=0.5, V(0)=0.3, I(0)=0.2, R(0)=0$.

We consider the parameters $\mu=0,03, \mu_{1}=0,04, \mu_{2}=0,035, \mu_{3}=0,06, \mu_{4}=$ $0,045, \beta=0,5, \beta_{1}=0,05, \gamma=0,1, \gamma_{1}=0,1, \alpha=0,02, \omega=0,1$ and the basic reproduction number is $R_{0}=1,17>1$. The endemic equilibrium point for this set of parameters are $(0.315,0.046,0.07,0.26)$. The numerical results are shown in Figure 3. 


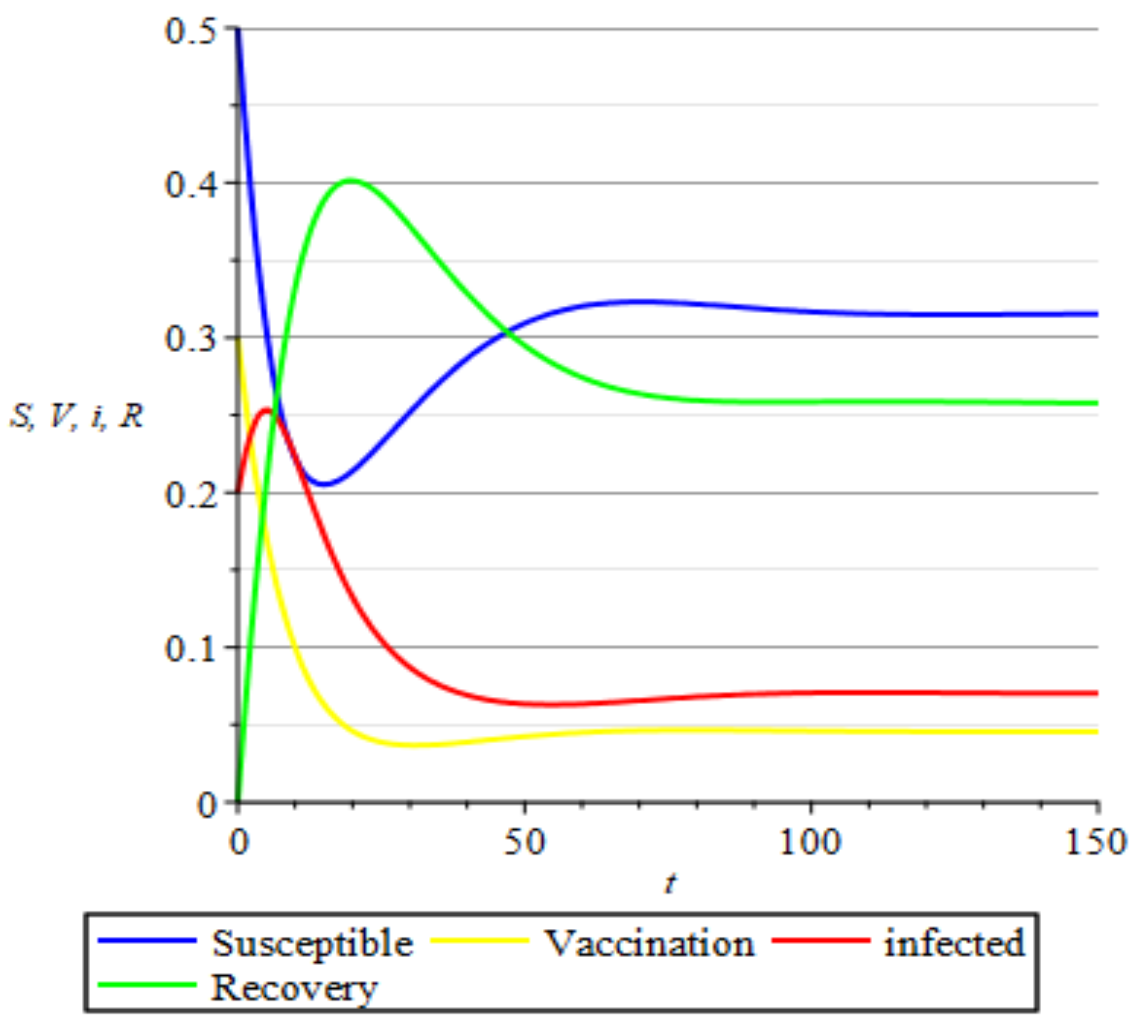

Figure 4. The dynamical behavior of system (1), when $R_{0}>1$ and the initial conditions are $S(0)=0.5, V(0)=0.3, I(0)=0.2, R(0)=0$.

\section{CONCLUSIONS}

From the results of endemic equilibrium point analysis, we define the basic reproduction number parameter, that is,

$$
R_{0}=\frac{\beta \mu}{\left(\alpha+\mu_{1}\right)\left(\mu_{3}+\gamma+\omega\right)}+\frac{\alpha \beta_{1} \mu}{\left(\alpha+\mu_{1}\right)\left(\gamma_{1}+\mu_{2}\right)\left(\mu_{3}+\gamma+\omega\right)}
$$

Furthermore, $R_{0}$ is a necessary condition for the existence of the two points of equilibrium as well as its local stability. When $R_{0} \leq 1$, there is only one (unique) equilibrium point of disease-free, that is, $E_{0}=\left(S_{0}, V_{0}, I_{0}, R_{0}\right)=\left(\frac{\mu}{\alpha+\mu_{1}}, \frac{\alpha \mu}{\left(\alpha+\mu_{1}\right)\left(\gamma_{1}+\mu_{2}\right)}, 0, \frac{\gamma_{1} \alpha \mu}{\mu_{4}\left(\alpha+\mu_{1}\right)\left(\gamma_{1}+\mu_{2}\right)}\right)$. Conversely, if $R_{0}>1$, then there are two equilibrium points, that is, $E_{0}$ and an endemic equilibrium point $E^{*}=\left(S^{*}, V^{*}, I^{*}, R^{*}\right)=\left(\frac{\mu}{\left(\alpha+\mu_{1}+\beta I^{*}\right)}, \frac{\alpha \mu}{\left(\alpha+\mu_{1}+\beta I\right)\left(\gamma_{1}+\mu_{2}+\beta_{1} I^{*}\right)}, I^{*}, \frac{\gamma_{1} V^{*}+\gamma I^{*}}{\mu_{4}}\right)$ where $I^{*}$ is the positive root of the equation $A_{1} I^{2}+A_{2} I+A_{3}\left(1-R_{0}\right)=0$.

Results of local stability analysis indicates if $R_{0}<1$, then the disease free equilibrium point $E_{0}$ is locally asymptotically stable. It means if the terms $R_{0} \leq 1$ fulfilled, then in a long time, there would have no spread of disease in susceptible and vaccinated subpopulations, or in other words, the epidemic will stop. Conversely, if $R_{0}>1$ then the endemic equilibrium point $E^{*}$ is locally asymptotically stable. It means, in a long time, the disease will always exist in the population with the proportion of each subpopulation is equal to $S^{*}, V^{*}, I^{*}$ and $R^{*}$. 


\section{REFERENCES}

[1] Diekmann,O., and Heesterbeek,J.A.P., Mathematical Epidemiology of Infectious Diseases: Model Building, Analysis, and Interpretation, John Wiley and Sons, Chichester, 2000.

[2] Driessche, P.V.D., Watmough, J., "Reproduction Number and Sub-threshod Endemic Equilibria for Compartmental Models of Disease Transmission," Mathematical Biosciences 180, pp. 29-48, 2002.

http://dx.doi.org/10.1016/s0025-5564(02)00108-6

[3] H.W. Hethcote, J.W.V. Ark, Epidemiological models for heterogeneous populations: proportionate mixing, parameter estimation, and immunization programs, Math. Biosci. 84 (1987) 85

[4] Kermack, W. O. and McKendrick, A. G., "A Contribution to the Mathematical Theory of Epidemics,"Proc. Roy. Soc. Lond., A 115, pp. 700-721, 1927. http://dx.doi.org/10.1098/rspa.1927.0118

[5] Khan, Muhammad Altaf, Saeed Islam and others, "Stability Analysis of an SVIR Epidemic Model with Non-linear Saturated Incidence Rate," Applied Mathematical Sciences, vol. 9, no. 23, .1145-1158, 2015.

http://dx.doi.org/10.12988/ams.2015.41164

[6] Kribs-Zaleta, C., Velasco-Hernandez, J., "A Simple Vaccination Model with Multiple Endemic State," Mathematical Biosciences, vol. 164, pp. 183-201, 2000.

[7] Lancaster, P.,"Theory of Matrices", Academic Press, New York, 1969.

[8] Liu, Xianing, and others, "SVIR Epidemic Models with Vaccination Strategies," Journal of Theoretical Biology 253, 1-11, 2007. http://dx.doi.org/10.1016/j.jtbi.2007.10.014

[9] Zaman, G., and others, "Stability Analysis and Optimal Vaccination of an SIR Epidemic Model," Bio Systems 93, pp. 240-249, 2008.

http://dx.doi.org/10.1016/j. biosystems.2008.05.004 